\title{
The American Board of Thoracic Surgery: Update
}

\author{
John H. Calhoon, MD, ${ }^{a}$ Richard J. Shemin, MD, ${ }^{b}$ Mark S. Allen, MD, ${ }^{c}$ and William A. Baumgartner, MD $^{d}$
}

\begin{abstract}
The primary purpose of the American Board of Thoracic Surgery (ABTS) is to protect the public by establishing and maintaining high standards in thoracic surgery. To achieve these objectives, the Board has developed qualifications for examination and procedures for certification and maintenance of certification. During the past few years, the ABTS initiated a new process for subspecialty certification in congenital cardiac surgery and a new process for maintenance of certification in accordance with requirements set forth by the American Board of Medical Specialties (ABMS), and collaborated with other organizations including the Residency Review Committee for Thoracic Surgery (RRC-TS), the Joint Council for Thoracic Surgical Education (JCTSE), and the Thoracic Surgery Directors Association (TSDA) to address some of the challenges currently facing our specialty. This report summarizes ABTS activities during the past year.
\end{abstract}

\section{PATHWAYS TO CERTIFICATION}

Eligibility to enter the ABTS certification process is currently achieved by completing 1 of the following 4 training pathways:

1. Completion of a general surgery residency (5 years) approved by the American Council on Graduate Medical Education (ACGME), followed by completion of a 2- or 3-year ACGME-approved thoracic surgery residency. Completion of an ACGME-approved Joint Training Program (a so-called 4/3 program) also fulfills this requirement. Certification by the American Board of Surgery (ABS) is optional for residents who started their residencies after July 1, 2003.

2. Completion of a general surgery residency or a cardiac surgery residency approved by the Royal College of Physicians and Surgeons of Canada, followed by successful completion of a 2- or 3-year ACGME-approved thoracic surgery residency.

From ABTS Chair, 2011-13, ${ }^{\mathrm{a}}$ University of Texas Health Science Center, San Antonio, Tex; ABTS Vice Chair 2011-13, ${ }^{\mathrm{b}}$ University of California-Los Angeles, Los Angeles, Calif; ABTS Examination Chair, 2009-14, ${ }^{\mathrm{c}}$ Mayo Clinic, Rochester,

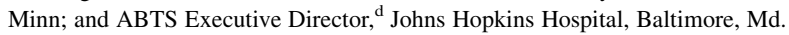

This article is being co-published in The Journal of Thoracic and Cardiovascular Surgery and The Annals of Thoracic Surgery.

Received for publication Feb 20, 2013; revisions received Feb 20, 2013; accepted for publication Feb 20, 2013.

Address for reprints: William A. Baumgartner, MD, Division of Cardiac Surgery, Johns Hopkins Hospital, $600 \mathrm{~N}$ Wolfe St, Blalock 618, Baltimore, MD 21287-4618 (E-mail: wbaumgar@jhmi.edu).

J Thorac Cardiovasc Surg 2013;145:1190-2

$0022-5223 / \$ 36.00$

Copyright (c) 2013 by The American Association for Thoracic Surgery and The Society of Thoracic Surgeons

http://dx.doi.org/10.1016/j.jtcvs.2013.03.008
3. Completion of an ACGME-approved vascular surgery residency that leads to eligibility for a primary certificate in vascular surgery, followed by completion of a 2- or 3-year ACGME-approved thoracic surgery residency.

4. Completion of an ACGME-approved integrated 6-year thoracic surgery residency (commonly termed, I-6 residency).

Recently, there has been increasing interest in the I- 6 residency pathway because it potentially allows residents to acquire knowledge and skills in thoracic surgery earlier in their training and also facilitates exposure to relevant allied disciplines in ways that other pathways do not. It is also hoped that this pathway will be a more attractive training paradigm to medical students and will help rectify the currently low number of applicants to thoracic surgical residencies. To date, there are 21 ACGME-approved I-6 programs. The number of applicants significantly outnumbers the available residency slots. To our knowledge, there has been only one resident, owing to health reasons, who dropped out of an I-6 program.

\section{REVISED OPERATIVE CASE REQUIREMENTS}

At the October 2011 meeting, the ABTS approved a revised set of operative case requirements for the final 2 to 3 years of thoracic surgical residency training. These revisions were developed in collaboration with the RRC-TS and took effect in July 2012. The new requirements reflect changes in the specialty (eg, requirements for minimally invasive lobectomy, endobronchial ultrasound, and catheter-based cardiac surgical procedures), add granularity to previous requirements, and add requirements for experience in critical care, simulation, and multidisciplinary patient care (eg, tumor boards or cardiac catheterization conferences). These requirements also were adopted to ensure every applicant regardless of pathway to certification had similar experiences. It is expected that these updated requirements will enhance the depth and breadth of residency experience.

\section{THE MILESTONE PROJECT}

The ABTS is collaborating with the Thoracic Surgery RRC in response to ACGME's new resident evaluation program. This will consist of milestones specific to cardiothoracic surgery, organized within the 6 core competences. The purpose of these milestones is to streamline the RRC program evaluation process, enhance ability to track resident progress, and clearly map progress to ABTSidentified curricular elements common to all certification pathways. These metrics will ultimately facilitate a shift 
from time-based to performance-based matriculation. The implementation of these milestones will occur with the entering class of residents in July 2014. They are currently being piloted within a few I-6 programs. There will be additional information available on the RRC and ABTS Web sites in the near future.

\section{NEW THORACIC SURGERY CURRICULUM}

Recognizing that operative case requirements constitute only 1 measure of thoracic surgical training, the ABTS collaborated with the JCTSE and the TSDA to create a thoracic surgery curriculum. Based on the curriculum developed in the United Kingdom, this document outlines in detail the cognitive and operative skills that every thoracic surgical resident should know upon completion of training. Not only does this curriculum provide guidance for residents and their program directors, it will also serve as the template for electronic teaching modules currently under development by the JCTSE and the TSDA. The Joint Council has recently completed populating this curriculum with ample content, which will soon be available to all thoracic residents in approved programs. Ultimately the Board will use this curriculum as a template for the content of both the written and oral examinations. A copy of the thoracic surgery curriculum can be found on the Board's recently revised Web site at www.abts.org.

\section{INITIAL CERTIFICATION EXAMINATIONS}

Overall pass rates for the initial qualifying examination declined during the period from 2003 to 2009, but began to increase in 2010 , with the rate of $85.4 \%$ in 2012 . While the pass rate on the qualifying (written) examination has improved, the pass rate on the certifying (oral) examination declined since 2007 when the ABTS changed the oral examination format to allow questions on a broader range of topics and to require that candidates pass both the cardiac and general thoracic components of the examination. The pass rates for the oral examination for 2011 and 2012 were $66 \%$ and $70 \%$ respectively, down from about $93 \%$ 10 years ago. Figure 1 depicts the pass results of the written and oral examinations over the last several years.

While it is possible that this trend reflects problems in the specialty as a whole with smaller numbers of residents (and therefore a less competitive pool of trainees) applying to thoracic surgical residencies, the ABTS is seriously concerned about this issue and has taken several steps to ensure that the examination is thorough, fair, and relevant to current practice. Like other ABMS Boards, the ABTS contracts with a psychometrician who determines the statistical validity of each examination and advises on changes in test format and content. In addition to rigorous annual review of all written and oral examination questions, the Board has intensified the orientation process for guest examiners (surgeons who are not ABTS Directors) and

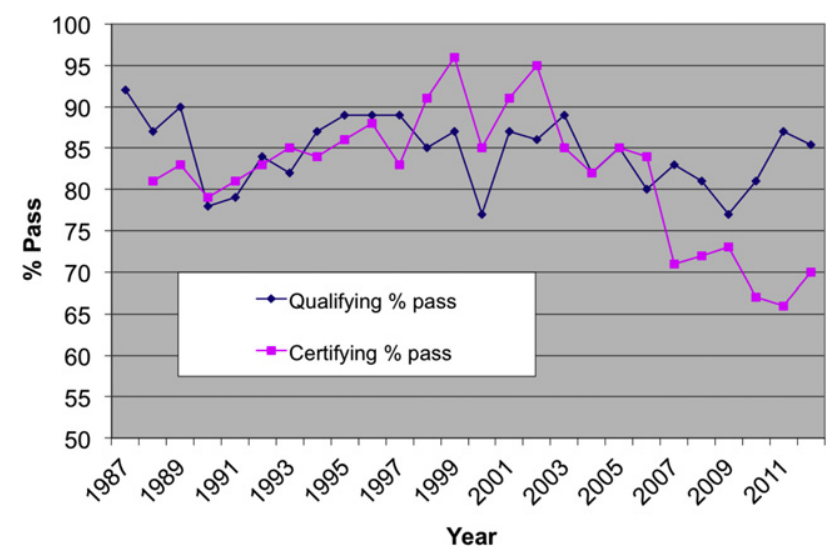

FIGURE 1. Pass results of the written and oral examinations for the last several years.

our Directors for the oral examination and recently developed an orientation video for candidates taking the certifying examination to familiarize them with the process (https://abts.org/oral_candidate_exam_orientation.htm). This is also available on the ABTS Web site.

During the past 3 years, the ABTS also instituted an annual process of soliciting nominations nationally from Division Chiefs in Cardiothoracic Surgery for examination consultants (who develop questions for the written examination) and oral examiners. This ensures that the ABTS draws upon the broadest possible range of expertise across the specialty. The declining examination pass rates were also a key reason the ABTS formalized guidelines for training by developing the thoracic surgical curriculum.

\section{CARDIOTHORACIC CRITICAL CARE EMPHASIS}

The ABTS recognizes cardiothoracic critical care as 1 of the 4 basic components of thoracic surgery along with adult cardiac surgery, general thoracic surgery, and congenital cardiac surgery, and has taken measures to emphasize this. As noted, documented experience in critical care will now be required to be eligible for initial certification. In addition, specific questions relating to critical care are now included in the initial certification and in the maintenance of certification examinations. The goal of these efforts is to ensure that ABTS Diplomates are prepared to care for their patients across the continuum of a hospital stay, including the intensive care unit, and have the necessary documentation to support doing so.

The ABTS has also promoted Critical Care as a career path for its Diplomates by collaborating with the ABS in the development of criteria for cardiothoracic-focused critical care fellowships that lead to subspecialty certification. Recent changes in the requirements for surgical critical care fellowships leading to certification by the ABS will now allow trainees to spend 4 to 6 months of a 1-year fellowship in a cardiothoracic surgical intensive care unit. Along with 
new requirements allowing more operative experience during critical care fellowships, these changes should make critical care an attractive training option for thoracic surgeons who wish to make this a career focus. Such fellowships can be undertaken as an additional year during I-6 residencies, or before or after the 2 to 3 years of training in traditional thoracic surgical residencies. Subspecialty certification is then possible through examination by the ABS once a primary specialty certification (either ABS or ABTS) is completed. The Board has considered supporting an independent certificate in Critical Care. Due to the lack of board-certified (both ABTS and Critical Care) academic thoracic surgeons as the educational core needed to apply for a separate certificate, the Board is not planning to pursue a separate certificate at this time.

\section{MAINTENANCE OF CERTIFICATION}

In response to an initiative by the American Board of Medical Specialties, the ABTS implemented its Maintenance of Certification (MOC) in January 2008. To date, approximately 1500 Diplomates have successfully participated in the MOC program, which now includes passing a secure examination. All Diplomates who are currently practicing, including those who hold lifetime certificates, are expected to participate in Parts I-IV of the MOC program. In addition, Diplomates who hold Inactive Status are expected to participate in Parts I-III. Diplomates must hold a valid and unrestricted ABTS certificate to enter the MOC process. The only pathway for renewal of a lapsed certificate is to take and pass the Part I (written) and the Part II (oral) certifying examinations. Detailed information is available on the ABTS Web site.

The current ABTS approach provides a comprehensive secure examination derived from Self-Education Self Assessment in Thoracic Surgery (SESATS). It has been associated with a high pass rate and general approval from Diplomates. The pass rate for the MOC examination has been over $95 \%$ in each of the 3 years since inception.
Similar to the certification examinations, the yearly MOC examination undergoes rigorous evaluation, led by a psychometrician, who determines the statistical validity of the examination.

\section{CONGENITAL CARDIAC SURGERY SUBSPECIALTY CERTIFICATION}

After several years of development leading to approval by the ABMS of a subspecialty certificate in Congenital Cardiac Surgery, the ABTS initiated the certification examination process in 2009. Two pathways currently permit certification in Congenital Cardiac Surgery, which can be obtained only after primary ABTS certification. "Pathway One" is the successful completion of a full congenital cardiac surgery residency approved by the ACGME, starting on July 1, 2008, or thereafter. "Pathway Two" is for those candidates who trained prior to July 1, 2008. The time to register for the examination was extended to 2014. Admission into the subspecialty certification process is based on training, current clinical experience, and professional accomplishments in the field. This pathway remains in effect only until 2014. To date 132 candidates have been certified in Congenital Cardiac Surgery. Seventy-three candidates have obtained certification via Pathway One and 125 candidates completed certification via Pathway Two. The process of MOC for Congenital Cardiac Surgery is now in development.

Rapid evolution in the practice of Thoracic Surgery during the past decade, new requirements for MOC, and problems in residency training have required the ABTS to become intimately involved in these areas because they affect certification in the specialty. A collaborative approach with the RRC-TS, JCTSE, TSDA, and ABS has been essential to addressing these challenges. As physicians come under increasing administrative oversight, continued dynamic involvement of the ABTS and regular reappraisal of the certification process is key to supporting the well-being of our specialty. 